\title{
LASER MICROIRRADIATION OF THE NUCLEOLAR ORGANIZER IN CELLS OF THE RAT KANGAROO (POTOROUS TRIDACTYLIS)
}

\author{
Reduction of Nucleolar Number and Production of Micronucleoli
}

M. W. BERNS, ${ }^{1}$ A. D. FLOYD,${ }^{2}$ K. ADKISSON, W. K. CHENG, L. MOORE, G. HOOVER, K. USTICK, S. BURGOTT and T. OSIAL

Departments of Zoology ${ }^{1}$ and Anatomy ${ }^{2}$, University of Michigan, Ann Arbor, Mich. 48104, USA

\begin{abstract}
SUMMARY
Cells of the established cell lines of the rat kangaroo (Protorous tridactylis), were laser microirradiated in order to study their nucleolar organizers. Microbeam irradiation of prophase and metaphase nucleolar organizer sites in both male (PTK 1) and female (PTK 2) cells resulted in a reduction in the number of normal nucleoli formed following mitosis. Irradiation of the one nucleolar organizer in the male cells, often resulted in the formation of numerous micronucleoli. Micronucleoli were also formed in small, extra nuclei, that were probably formed as a result of microirradiation of the centromere region of autosomes. Preliminary cytochemical analysis of the micronucleoli is presented and the possible mechanism of the formation is discussed.
\end{abstract}

Cells from the established lines of the rat kangaroo (Potorous tridactylis) are particularly useful in studies employing microbeam irradiation because of their low chromosome number, and because they remain flat during the mitotic process. Both of these characteristics facilitate microbeam irradiation of a desired chromosome [1].

A nucleolar-associated secondary constriction on the " $\mathrm{X}$ " chromosome has been noted by several authors [2, 3], and examined ultrastructurally by Hsu et al. [4]. The occurrence of the secondary constriction on an easily recognizable chromosome suggests that it might be irradiated with the laser microbeam. Initial studies on metaphase and anaphase salamander cells demonstrated that laser microbeam irradiation of secondary constrictions, or irradiation of the chromosome re- gion immediately adjacent to the constriction, resulted in the loss of nucleolar synthesis by the irradiated chromosome $[5,6]$. Subsequent studies involving microirradiation of nucleolar associated chromosomes during prophase also resulted in a reduction of nucleolar number [7]. The salamander cells normally contained two, three or four nucleolar-associated secondary constrictions, with the same number of associated nucleoli. When either one or two of the nucleolar organizers were destroyed, the nucleoli that were formed (either two or three) were considerably larger than normal. There appeared to be a compensation mechanism similar to the phenomenon described in the heterozygous anucleolate Xenopus mutant [8].

A highly desirable microbeam experiment would have been the elimination of all the 
major nucleolar organizers from the cell. This experiment was not possible because of the difficulty in finding and irradiating all of the nucleolar organizers in the same cell during the appropriate stages of mitosis. The availability of both male (PTK 2) and female (PTK 1) cell lines of the rat kangaroo presents several interesting possibilities for the study of the nucleolar organizer. The male cell line (PTK 2) contains only one X chromosome per cell, one secondary constriction, and most of the cells have only one nucleolus. The female cell line (PTK 1 ) contains two $\mathrm{X}$ chromosomes per cell, two secondary constrictions, and most frequently two nucleoli per cell. It would, therefore, seem feasible to selectively delete the one secondary constriction from the male cell and either one or both secondary constrictions from the female cells. Assay of nucleolar formation following the preceding experimental manipulation would help clarify the relationship between the secondary constriction and the nucleolus. In addition, the removal of a nucleolar organizer from the male cells (PTK2) would result in a cell completely lacking its major nucleolar organizer. Would these cells survive and produce normal nucleoli?

\section{MATERIALS AND METHODS}

The male (PTK 2) and female (PTK 1) cell lines were obtained from the American Type Culture Collection, Washington, D. C. Stock cells were maintained in T60-like flasks and subcultured once weekly. Cells were grown in minimal essential Eagle medium, with Earle salts, sodium pyruvate $(0.11 \mathrm{~g} / \mathrm{l})$, sodium bicarbonate $(0.85 \mathrm{~g} / \mathrm{l}), 10 \%$ fetal calf serum; 100000 IU penicillin and $0.05 \mathrm{~g} / \mathrm{l}$ streptomycin. $\mathrm{pH}$ was adjusted to 7.0. Two to four days prior to an irradiation experiment cells were trypsinized $(0.125 \%$ trypsin) from the stock flasks, resuspended in culture medium and seeded in Rose multipurpose culture chambers. Immediately preceding irradiation, cells were treated for $5 \mathrm{~min}$ with a solution of $0.001 \mu \mathrm{g}$ of acridine orange per $\mathrm{ml}$ of culture medium. After two washes with culture medium, fresh medium was placed in the chamber and the irradiation performed.

Cells were irradiated either in prophase or metaphase. Prophase cells were irradiated at the chromo- some sites that appeared to be associated with the nucleolus. In the female cells only one of the two nucleoli was irradiated at its chromosome-associated site. Male cells were irradiated at the chromosome sites of the single nucleolus. Irradiation was performed when the nucleolus began to disappear and the chromosomes were moderately condensed. Metaphase irradiation was performed only at the secondary constriction region of the male cells.

The microbeam system has been described in detail elsewhere [9]. The beam of an argon ion gas laser (Hughes $3030 \mathrm{H}$ ) with primary wavelengths at 488 $\mathrm{nm}$ and $514 \mathrm{~nm}$ was directed into a standard Zeiss Photomicroscope and focused by a Zeiss Neofluar $\times 100$ oil immersion objective (N.A. 1.30). A television camera and monitor was attached to the microscope and provided a continual image of the specimen. Irradiation was performed by locating the image of the cell on the screen of the TV monitor and moving the target area directly under a crosshair on the TV screen. Pre-alignment assured that the cross-hair denoted the focal point of the laser beam. In all experiments a focal spot diameter of between 0.5 and $1.5 \mu \mathrm{m}$ was used. Energy density in the focal spot varied from $30-40 \mu \mathrm{J}$. Pulse duration was $50 \mu \mathrm{sec}$. The total amount of energy delivered to each cell varied, depending upon the number of chromosomes irradiated. The range of total radiation exposures was 300-800 $\mu \mathrm{J}$.

Following irradiation nucleolar formation was observed and photographed. Several cells were fixed and stained and the nucleoli cytochemically analysed. Nucleolar RNA was asayed by staining with cresyl violet according to the procedures of Ritter et al. [10]. Following fixation in acetic acid/formol/70\% ethanol $(1: 1: 20)$ for $20 \mathrm{~min}$ cells were rinsed in a $70 \%$ ethanol and air dricd for starage. Staining was for $2 \mathrm{~h}$ in $0.1 \%$ aqueous cresyl violet at $45^{\circ} \mathrm{C}$ and pH 4.2. Differentiation was done in absolute ethanol for $24 \mathrm{~h}$, followed by two washes in fresh absolute ethanol, cleared in xylene and mounted in permount. Nucleolar protein was stained by the Naphthol yellow procedure of Deitch [11]. Cells were fixed and stored as above. Staining was performed in a solution of $0.01 \%$ naphthol yellow $\mathrm{S}$ in $1 \%$ acetic acid for $1 \mathrm{~min}$. Differentiation was in two changes of $1 \%$ acetic acid, 2 min in each. Cells were dehydrated in two changes of tertiary butyl alcohol, $3 \mathrm{~min}$ in each, passed into xylene, and mounted in Permount.

\section{RESULTS}

\section{Normal karyology and nucleoli}

A karyotype of the PTK 1 and PTK 2 cell lines is illustrated in fig. $1 a, c$. The two secondary constrictions of the female cell line, and the single constriction of the male cell line are indicated. The corresponding nucleolar number of both cell lines are shown in fig. $1 b, d$. Most of the female cells contain 


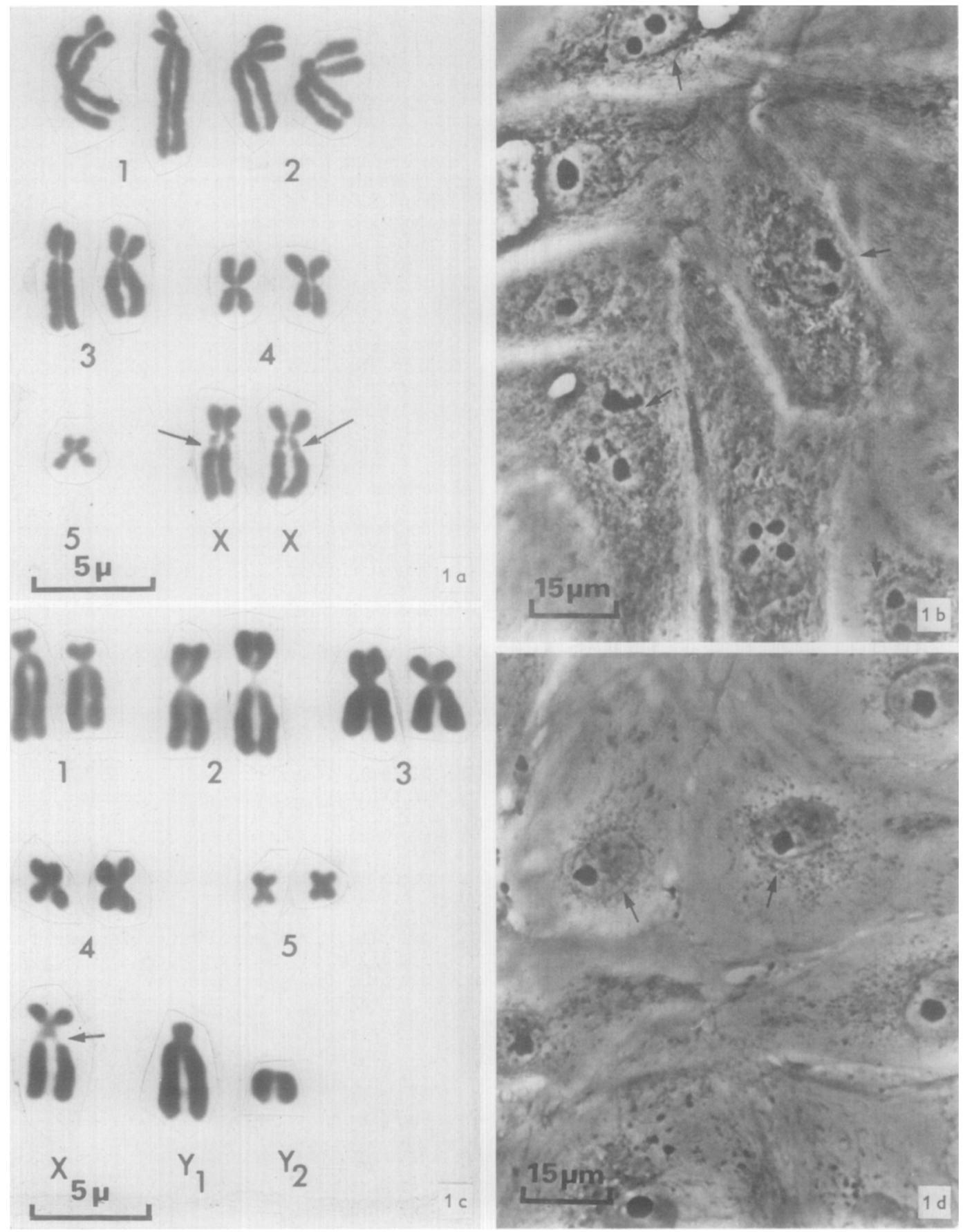

Fig. 1. (a), female, PTK 1 cell karyotype; note the two X chromosomes with secondary constrictions (arrows); (b) female cells in interphase; note most cells have two nucleoli; (c) male, PTK 2 cell karyotype; note the one $\mathrm{X}$ chromosome with a secondary constriction: phase optics; $(d)$ male interphase cells; note one nucleolus per cell; phase optics.

Exptl Cell Res 75 (1972) 

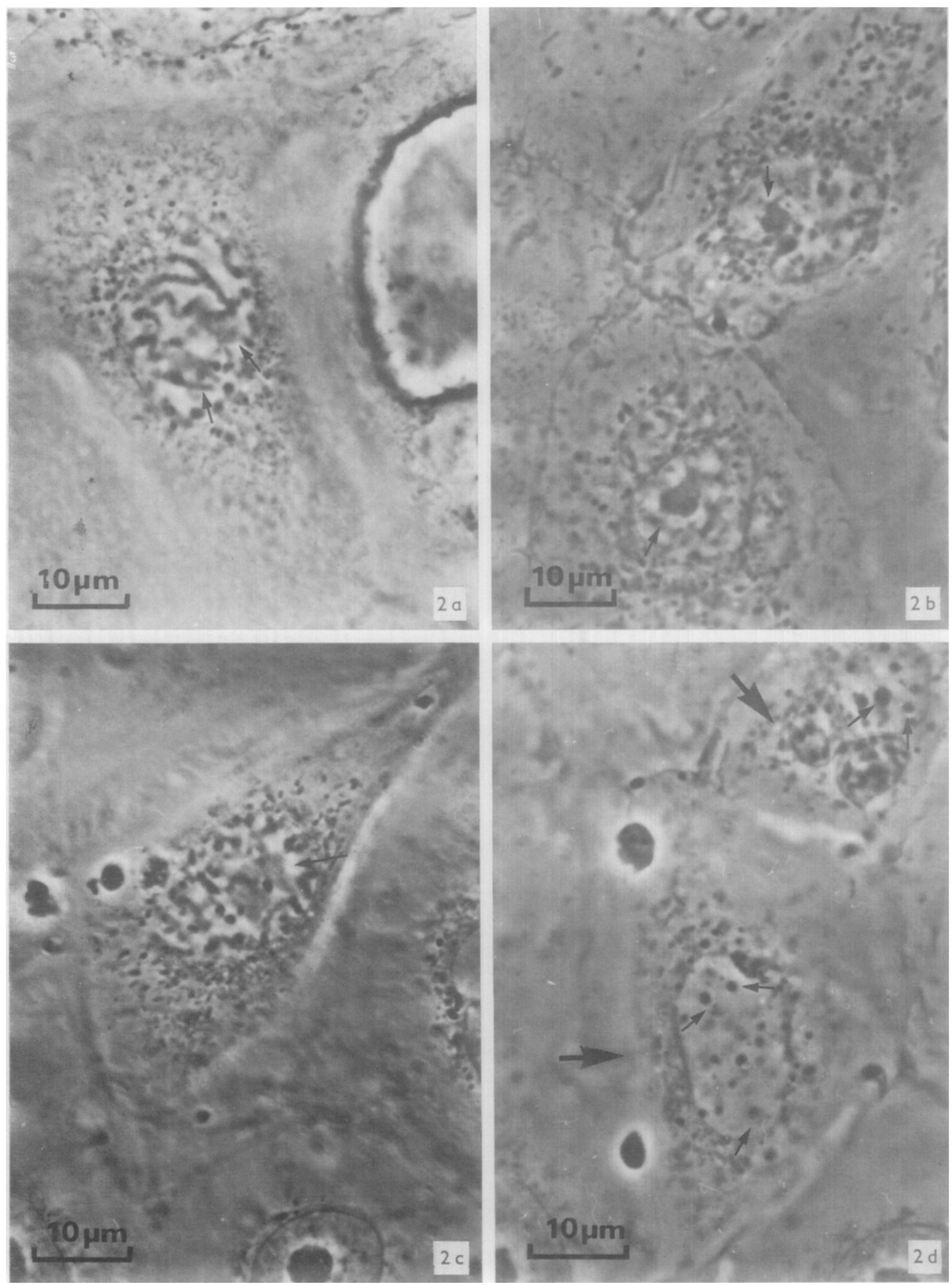

Fig. 2. (a) middle prophase of female (PTK 1) cell; note the two nucleoli (arrows) and the chromosomes associated with the nucleoli; this cell was irradiated at the chromosome regions near the nucleoli immediately after this photo was taken; $(b)$ two daughter cells formed from the preceding cell; note only one nucleolus per cell (arrows); chromatin has not fully decondensed yet; phase optics one hour post-irradiation; $(c)$ male (PTK 2) cell in middle prophase immediately preceding microirradiation of nucleolar-associated chromosomes; note single nucleolus (arrow); (d) two daughter cells formed from preceding cell (large arrows); note numerous small micronucleoli in each nucleus (small arrows); phase optics $2 \mathrm{~h}$ post-irradiation. 

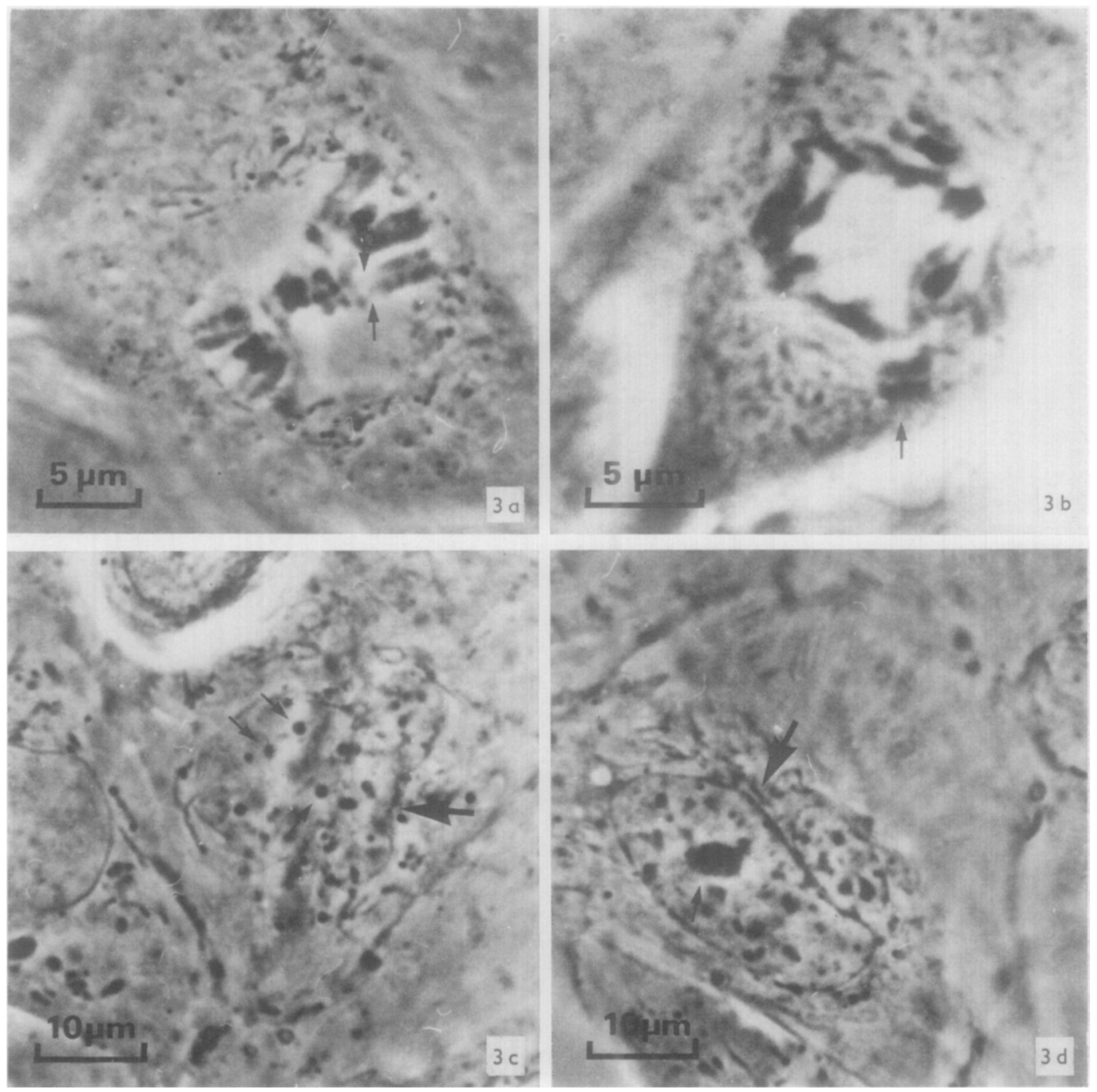

Fig. 3. (a) male (PTK 2) cell in metaphase after microirradiation of the secondary constriction region on the X chromosome; both X chromatids were irradiated; note the "paled" spots (arrows); $(b)$ anaphase of preceding cell; note the chromosome(s) apparently unattached to the spindle (arrow); phase optics 20 min postirradiation; (c) one of the daughter nuclei of preceding cell (large arrow); note the numerous small micronucleoli (small arrows); $(d)$ other daughter cell with two nuclei (large arrow); one nucleus has large nucleolus (arrow), and other smaller nucleus (apposed to top right of large nucleus), has several small micronucleoli, phase optics $24 \mathrm{~h}$ post-irradiation.

two nucleoli and most of the male cells have one nucleolus. Both cell lines do contain some cells with abnormal numbers of nucleoli. The PTK 1 cell line is generally monosomic for chromosome 5 .

\section{Prophase irradiation of female PTK1 cells}

Irradiation of the nucleolar associated chromosome sites of one of the nucleoli in a two nucleolar cell is depicted in fig. $2 a, b$. The 

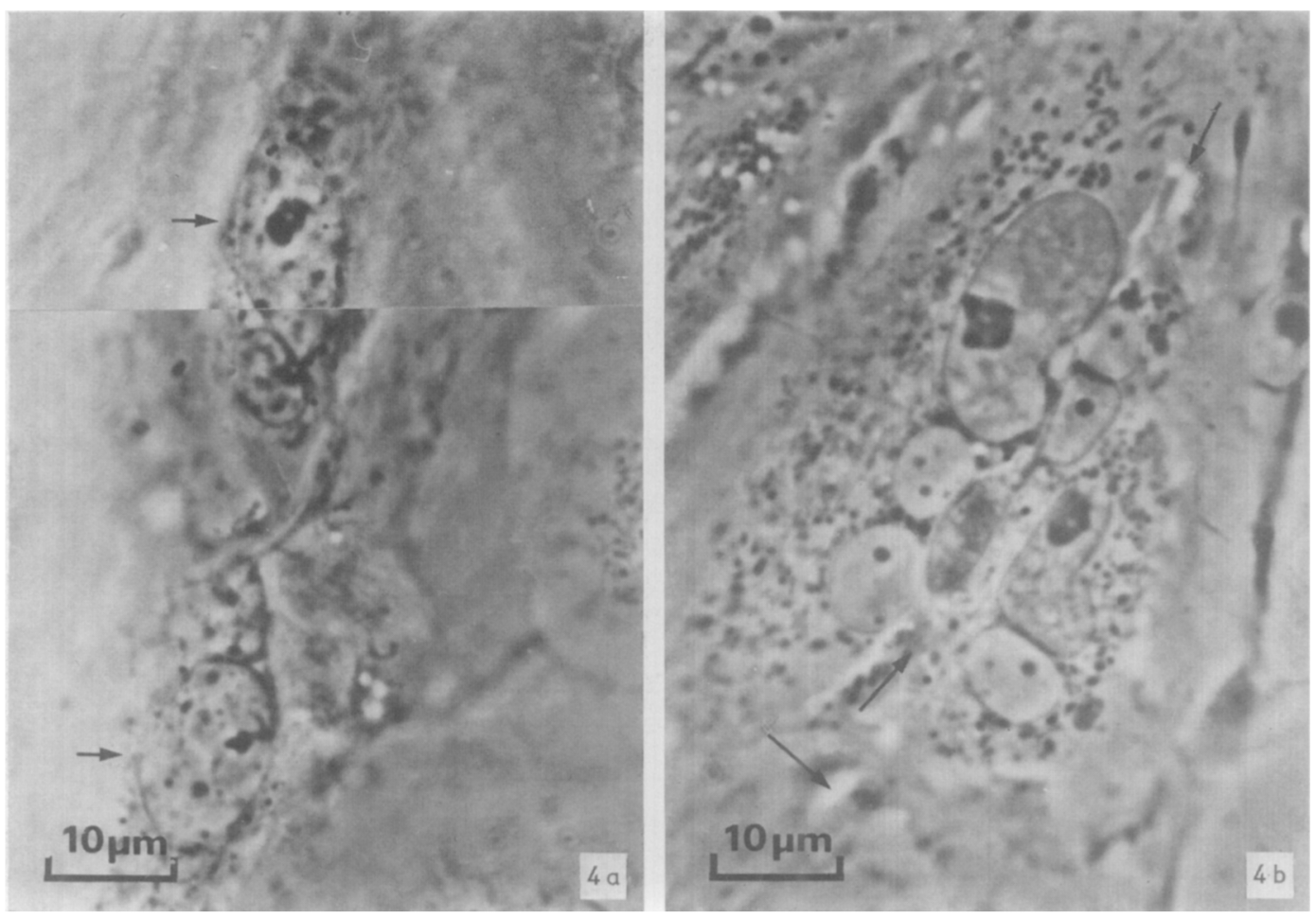

Fig. 4. (a) two daughter cells (arrows) resulting from metaphase microirradiation of secondary constrictions of male (PTK 2) cells; top cell has normal large nucleolus, and bottom cell has numerous micronucleoli; phase optics $24 \mathrm{~h}$ post-irradiation $(b)$ the result of probable microirradiation of autosome in centromere region; large arrows denote margin between two closely aposed cells; both cells have a nucleus with a single large nucleolus, and small nuclei with micronucleoli; phase optics $24 \mathrm{~h}$ post-irradiation.

cell completed mitosis and formed two daughter cells with one nucleolus in each cell. 'This experiment was repeated three times with the same results.

\section{Prophase irradiation of male PTK2 cells}

The chromosomes associated with the single nucleolus were irradiated as illustrated in fig. $2 c, d$. The cell completed mitosis and two daughter cells were formed. Numerous small nucleolar-like bodies (micronucleoli) were produced in each daughter cell nucleus. This cell was followed for $48 \mathrm{~h}$ in culture. Similar results have been observed in three repeats of the experiment. None of the micronucleoli-containing cells were observed entering into subsequent mitosis.

\section{Metaphase irradiation of male cells}

The results of the metaphase irradiations were less clear cut than the previous experiments. In one experiment irradiation of the secondary constriction region of the $\mathrm{X}$ chromosome resulted in two daughter cells: one daughter cell had a single nucleus containing only micronucleoli (fig. $3 c$ ), and the other daughter cell had two nuclei-a large one with a normal appearing nucleolus, and a smaller nucleus with several small micronucleoli (fig. $3 d$ ). As can be seen from fig. $3 b$, some chromosomes or chromosome fragments failed to undergo normal anaphase movements and segregation.

In another experiment, abnormal chromosome segregation (chromosomes did not 


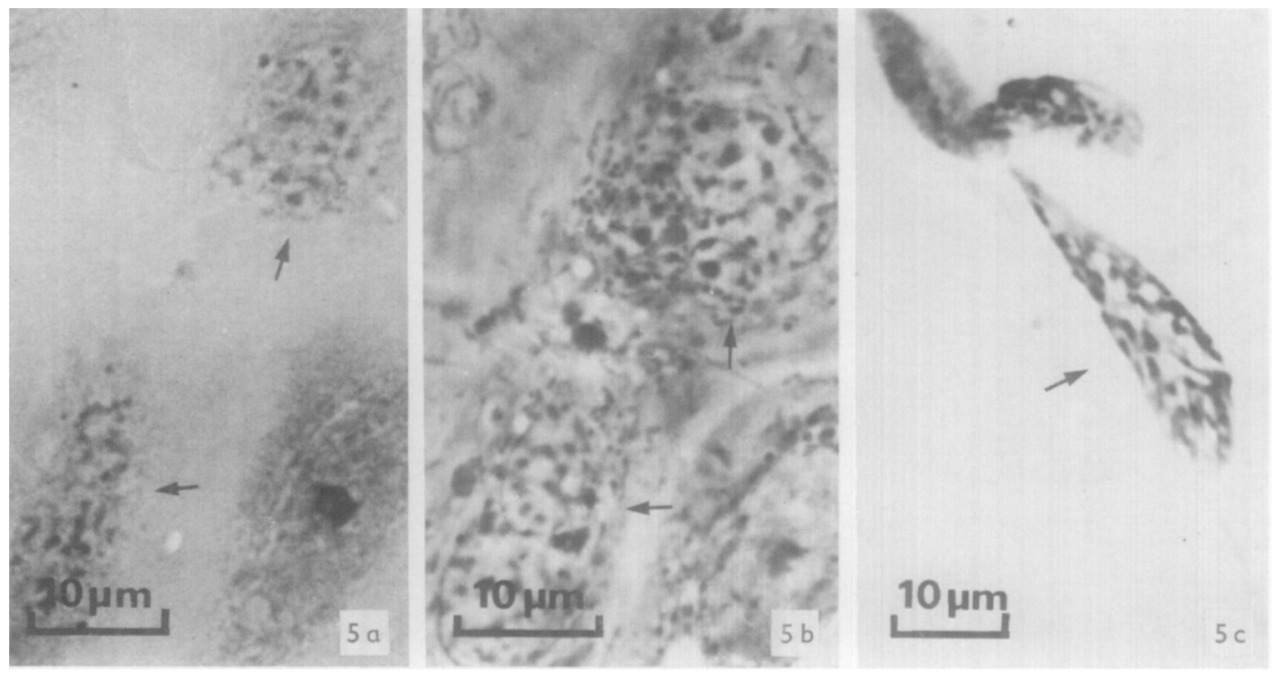

Fig. 5. (a) two daughter cells (PTK 2) with micronucleoli positively stained with cresyl violet for RNA; (b) same cells prior to fixation under phase optics; these cells were formed following irradiation similar to that described in fig. $2 c, d ;(c)$ cell with micronucleoli positively stained for protein with naphthol ycllow.

appear to be attached to the mitotic spindle) also occurred and the result was one cell with micronucleoli and another cell with a normal nucleolus (fig. $4 a$ ).

In a third experiment, irradiation of what we thought was the $X$ chromosome but probably was an autosome, resulted in abnormal chromosome movement. The result was two daughter cells with several nuclei. In both cells, one nucleus contained a normal looking nucleolus, and the other nucleus contained micronucleoli (fig. $4 b$ ).

\section{Cytochemical analysis}

Micronucleoli stained positively for both RNA and protein. Fig. $5 a$ is a cell stained for RNA with cresyl violet, and fig. $5 c$ is a cell stained for protein with naphthol yellow. In fig. $5 b$, the cell stained with cresyl violet is shown through the phase microscope prior to fixation. These are PTK2 (male) cells that have been irradiated in a fashion identical to those cells described in the previous section "Prophase irradiation of male PTK 2 cells”.

\section{DISCUSSION}

The morphology and karyology of the rat kangaroo cells make them ideal for cytogenetic and microbeam studies. The location of nucleolar associated secondary constrictions on the $\mathrm{X}$ chromosome provides a sex-linked characteristic that is expressed biochemically and morphologically in cultured cells. The availability of stable male and female cell lines with one and two secondary constrictions respectively, and corresponding nucleolar number ( 1 per constriction) is a unique occurrence in cultured cells. In addition, it appears that $\mathrm{X}$-chromosome inactivation (Lyon's Hypothesis) does not occur in the female cells (PTK 1)--at least with respect to the nucleolar genes. One wonders if this is also the case in vivo.

The reduction of one nucleolar organizer and concomitant decrease in nucleolar number in the female cells agrees with the experiments performed on primary cultures of salamander lung $[5,6]$. However, the ability to perform this manipulation in established 
cell lines provides the capability of establishing new cell lines deficient in varying numbers of nucleolar organizers. It should be possible to clone these cells into a viable cell strain. It has already been demonstrated that microirradiated rat kangaroo ceils can undergo additional mitosis [1].

The occurrence of micronucleoli is a phenomenon described by many authors. Small nucleolar-like bodies have been found associated with dipteran polytene chromosomes [12, 13]. Garcia \& Kleinfeld [14] have demonstrated that they are active in RNA synthesis. McClintock [15] and Heitz [16] showed that when the major nucleolar organizer was absent from the chromosome of Zea mays, micronucleoli were produced at other chromosome sites. Phillips \& Phillips [17] have described numerous micronucleoli in cultured Chinese hamster cells. These bodies occurred in normal nuclei and in micronuclei produced by treating the cells with colchicine. Cytochemical analysis of the micronucleoli indicated they contained both RNA and protein. Radioautography indicated that they were capable of RNA synthesis, and electron microscopy demonstrated that the micronucleoli contained fibrillar and granular components.

Abnormal micronucleoli have been experimentally induced in Chinese hamster embryonal cells, KBG human cells, and C929 mouse cells by treatment with actinomycin $\mathrm{D}$ for $3 \mathrm{~h}$ prior to mitosis. Following mitosis, small nucleolar-like refractile bodies formed that stained positively for protein, and negatively for RNA (Phillips. In press). On the other hand, Barr [18] has discussed micronucleoli ('blobs') from the anucleolate Xenopus mutant that stain positively for both RNA and protein.

It is evident that micronucleoli vary with respect to structure and function, depending upon the organism and the method of pro- duction. The micronucleoli formed in male rat kangaroo cells as a result of deleting the major nucleolar organizer during prophase appear to contain both RNA and protein. A logical explanation for their occurrence would be the presence of additional 'subsidiary' nucleolar organizer sites that were activated when the major nucleolar organizer was deleted. This mechanism could also explain why deletion of one major nucleolar organizer from the female cells did not result in micronucleoli; the remaining major nucleolar organizer could still organize a normal nucleolus.

An alternative hypothesis would be that the micronucleoli are formed from nucleolar proteins and RNA that were synthesized prior to mitosis (Phillips. In press).

The experiments involving metaphase irradiation of male cells are more complicated. In all three of the experiments described at least one of the two daughter cells contained a nucleus with a large normal-appearing nucleolus, and a nucleus with micronucleoli. In addition, abnormal anaphase chromosome movements were observed in all three experiments. The abnormal chromosome movments could be explained by the fact that in the fully condensed metaphase chromosome, the secondary constriction is situated very close to the centromere (see fig. $1 a$ ). It is possible that, in attempting to irradiate the secondary constriction, the centromere was damaged. This could result in the inability of the irradiated chromosome to attach to the spindle and segregate normally. Chromosomes could end up not segregating at all, moving into the wrong cell nucleus or being excluded from the major nucleus, thus resulting in the formation of a micronucleus. In addition, the metaphase irradiation could result in damage to varying combinations of secondary constrictions and/or centromeres. 
In summary, it appears possible to produce micronucleoli by either direct microirradiation of the single major nucleolar organizer of the $\mathrm{X}$ chromosome of male cells, or by irradiation of the centromeres. Irradiation of the $\mathrm{X}$ chromosome centromere can result in the inclusion of the $\mathrm{X}$ chromosome in the wrong nucleus or into a small micronucleus. Micronucleoli are formed in the nucleus deficient in the $\mathrm{X}$ chromosome. It also secms possible to produce micronucleoli by irradiating the centromeres of autosomes (not $X$ chromosomes). This type of irradiation results in the exclusion of the irradiated chromosome from the normal nucleus and its inclusion into a micronucleus. This is probably what happened in the experiment illustrated in fig. $4 b$. The fact that a large nucleolus was produced in two of the nuclei indicates that the secondary constrictions probably were not irradiated. The irradiated chromosomes were probably autosomes that were segregated into separate nuclei. When isolated from the major nucleus which contains the primary nucleolar organizer the secondary nucleolar organizers on the autosomes were able to function. This result would suggest that in the male rat kangaroo, ribosomal cistrons are distributed between many chromosomes. These genes normally are repressed, but are expressed when the major group of ribosomal cistrons are inactivated or isolated in another nucleus. This hypothesis is similar to the one suggested by Phillips \& Phillips [17] for the Chinese hamster.

This work was supported in part by the following grants: NSF GB 24457; NIH HE 13750; Amer. Cancer Soc. IN40K and IN40L; University of Michigan, Phoenix, Rackham, and IST grants.

\section{REFERENCES}

1. Berns, M W, Cheng, W K \& Hoover, G, Nature (London) 233 (1971) 122.

2. Walen, K H, Genetics 51 (1965) 915.

3. Shaw, M \& Krooth, G, Cytogenetics 3 (1964) 19.

4. Hsu, T C, Brinkley, B R \& Arrighi, F E, Chromosoma (Berlin) 21 (1967) 137.

5. Berns, M W, Ohnuki, Y, Rounds, D E \& Olson, R S, Exptl cell res $60(1970) 133$.

6. Berns, M W \& Cheng, $\mathrm{W} \mathrm{K}$, Exptl cell res 69 (1971) 185.

7. Ohnuki, Y, Rounds, D E, Olson, R S \& Berns, M W, Exptl cell res 71 (1972) 132 .

8. Barr, H J \& Esper, H, Exptl cell res 31 (1963) 211.

9. Berns, M W, Exptl cell res 65 (1971) 470.

10. Ritter, C, Distegano, M S, Farah, R, J histochem cytochem 9 (1961) 97.

11. Deitch, A D, J histochem cytochem 13 (1965) 17.

12. Swift, $H$, The molecular control of cellular activity (ed J M Allen) p. 73. McGraw Hill, New York (1962).

13. Jacob, J \& Sirlin, J L, J cell biol 17 (1963) 153.

14. Garcia, N G \& Kleinfeld, R G, J cell biol 29 (1966) 347.

15. McClintock, B, Z Zellforsch mikroskop Anat 21 (1934) 294.

16. Heitz, E, Planta (Berlin )12 (1931) 775 .

17. Phillips, S G \& Phillips, D M, J cell biol 40 (1969) 248.

18. Barr, H J, Natl cancer inst monograph 23 (1966) 411 .

Received April 19, 1972

Revised version received May 23, 1972 